\title{
The Simulation Training System design program evaluation of communication equipment
}

\author{
Ping Gong, Xingcun WU
}

China Satellite Maritime Tracking \& Controlling Department, Jiangsu Jiangyin 214400, China.

Keywords: Fuzzy theory, Fuzzy comprehensive evaluation, Simulation training system

\begin{abstract}
According to the need to establish simulation training system of communication equipment, the paper proposes using fuzzy comprehensive evaluation method for quantitative evaluation of each alternative design program. Finally put forward the optimal design program.
\end{abstract}

\section{Introduction}

With the information level of communication equipment continue to improve, limited system hardware and software, support capability of communication equipment is not strong enough, the application of simulation training systems for training has become a routine way of training is also an important way of training. Simulation training can reduce training costs and reduce loss of communication equipment, which can be played back and commenting after recording the training data, reproducing equipment status and operational details, and conditions of the training not subject to time and space restrictions and climatic. Build efficient communication equipment support simulation training system can have important significance to enhance communication equipment support capabilities.

The restricting factors of communication equipment simulation training system design involves technical, capital, the demand for units, logistics force, research and development cycle etc. The merit of the design directly determines the quality of the system, and uncertainty of many other factors is large, multiple fuzzy concept. Therefore, in solving this problem, select multi-level Fuzzy comprehensive evaluation method, you can select the optimal design program in many alternatives.

\section{Fuzzy Comprehensive Evaluation}

Fuzzy comprehensive evaluation method is to analysis of the numerical merits of the program through the establishment of an evaluation index system, given the reviews membership of target layer. The method that is given assessment of the merits by the size of the numerical, and it is reliable and effective method to evaluate the fuzzy and relative index.

\subsection{Fuzzy Comprehensive Evaluation Model}

To properly judge a specific object, firstly we should gives reviews the factors of this object, and then integrated. Factor is the various properties or performance of the object, on different occasions, also known as parameter indicators or quality indicators, which can reflect the quality of the object integrated, so these factors can be evaluated. General step of fuzzy comprehensive evaluation model is:

1) Establish the evaluation factors, that is a collection of the various factors that influence the evaluation objects,

$$
U_{i}=\left\{U_{i 1}, U_{i 2}, \cdots, U_{i n}\right\}
$$

2) Establishment the evaluation set.

$$
V=\left\{v_{1}, v_{2}, \cdots, v_{n}\right\}
$$

3) Establish single factor judge, that is to build a Fuzzy mapping from $U$ to $F(V)$,

$$
\gamma: U \rightarrow F(V) . u_{i} \rightarrow \gamma\left[u_{i}\right]=\frac{\gamma_{i 1}}{v_{1}}+\frac{\gamma_{i 2}}{v_{2}}+\cdots+\frac{\gamma_{i m}}{v_{m}}
$$


We can induct the Fuzzy relations by $\gamma$ and then obtain Fuzzy matrix,:

$$
R=\left[\begin{array}{cccc}
\gamma_{11} & \gamma_{12} & \cdots & \gamma_{1 n} \\
\gamma_{21} & \gamma_{22} & \cdots & \gamma_{2 n} \\
\vdots & \vdots & \cdots & \vdots \\
\gamma_{m 1} & \gamma_{m 2} & \cdots & \gamma_{m n}
\end{array}\right]
$$

Which is called single factor evaluation matrix of $R$, and the triple orderly $\operatorname{group}(U, V, R)$ call evaluation space.

4) Multi-level fuzzy comprehensive evaluation. Evaluate start with the underlying property index to the top index layer by layer. We can obtain the comprehensive evaluation vector of sub-target $X_{k}$,namely $B_{k}=A_{k} \cdot R_{k}$, which conduct fuzzy matrix merge operation by the attribute index weight vector $A_{k}$ of sub-target $X_{k}$ and single factor fuzzy evaluation matrix $R_{k}$. Then obtain a single factor fuzzy evaluation matrix, finally it is concluded the comprehensive evaluation result of the target layer.

5) Select the algorithm. Commonly used algorithms mainly by weighted average operator, geometric average operator, single factor determine operator and the type of the primary factors highlight operator. Judge issues involved in dealing with many factors, such as communications equipment simulation training system involves many evaluation factors, the merits of the design program is a comprehensive reflection of a variety of factors, generally select weighted average operator.

Let $A=\left\{a_{1}, a_{2}, \cdots, a_{n}\right\} \in[0,1]^{n}$ be the normalized right amount, $\forall\left(x_{1}, x_{2}, \cdots, x_{n} \in[0,1]^{n}\right)$ and $\mathrm{f}_{\Sigma}=\left(x_{1}, x_{2}, \cdots, x_{n} x_{n}\right)=\sum_{i=1}^{n} a_{i} \mathrm{x}_{\mathrm{i}}, f_{\Sigma}$ is called weighted average Fuzzy comprehensive functions. Which $a_{i}$ is the $i$-th factor in proportion of comprehensive evaluation.

6) Evaluate merits of the training system design program. When the training system design program is only one, we can judge on the merits of the program according to evaluation grades corresponding to the maximum membership degree of the last judgment vector $B$. When there are more than one design program, we can use the following method to quantify the evaluation results, which can be horizontal comparison, select the best design program.

First, give the judge level a quantization value: $V_{1}=100, V_{2}=75, V_{3}=50, V_{4}=25$, then, do normalized processing for evaluation vector $B$ using the following formula: $E=B \cdot V=\sum_{i=1}^{4} b_{i} v_{i}$, lastly, according to the size of $E$ can visually judge what kind of programs Better.

\section{2 determine the index factor weights}

1) Establish the evaluation index system.

2) Quantify all levels of criteria using the 1: 9 ratio scaling method to obtain judgment matrix $C$.

3) Calculate the maximum eigenvector $\omega$ and maximum eigenvalue $\lambda$. Usually you can calculate using the square root method, where $\overline{\omega_{i}}=\sqrt[n]{\prod_{j=1}^{n} a_{i j}}, i=1,2, \mathrm{~L}, n$. Standardized $\overline{\omega_{i}}$ to get $\omega_{i}$, $\omega_{i}=\frac{\overline{\omega_{i}}}{\sum_{i=1}^{n} \overline{\omega_{i}}}, i=1,2, \mathrm{~L}, n, \omega_{i}$ is the $i$-th component of $\omega$. Finally calculate $\lambda_{\max }, \lambda_{\max }=\sum_{i=1}^{n} \frac{\sum_{i=1}^{n} a_{i j} \omega_{i}}{n \omega_{i}}$.

4) Consistency test.

In order to prevent the emergence of inconsistencies in judgment, we also need to test using the 
consistency index C.I when we obtain judgment matrix $C$, which $C . I=\frac{\lambda_{\max }-n}{n-1}$. Generally it can be considered to be judgment matrix $C$ satisfactory as long as $C . I \geq 0.1$.

\section{Alternatives design program presentation of communication equipment simulation training system}

There are mainly four kinds of design program of simulation training system: physical simulation training systems, semi-physical + digital technology simulation training system, based on visual simulation technology training systems, based on virtual reality technology simulation training systems. Among them, the first kind of program of the simulation training systems is based on the operation of physical, which can achieve the same training effect liking the actual equipment, but the development cycle is long and the investment cost is high. The investment cost of the second kind of program is low and the development cycle is short, which realization technology is relatively simple, but the training effect is poor. The third program is mainly focused on the training of visual effects which has better application in simulated operation of large-scale communications equipment. The fourth program has good simulation training effects, which even can achieve better training effect than real equipment, but the investment cost is high, and the realization technology is complex, development cycle is long, equipment maintenance is difficult. In summary, each program has its own advantages and disadvantages, it must be quantitatively evaluated to choose a suitable design of the system.

\section{Design program evaluation of communication equipment simulation training system}

We can evaluate the merits of the communication equipment simulation training system design program according to the fuzzy comprehensive evaluation method described above. There are four kinds of options: (1) physical; (2) semi-physical + digital technology; (3) Technology of simulation training systems based on visual; (4) Technology of simulation training systems based on Virtual Reality (VR).

Firstly establish the evaluation index system (evaluation factor set), shown in Figure 1.

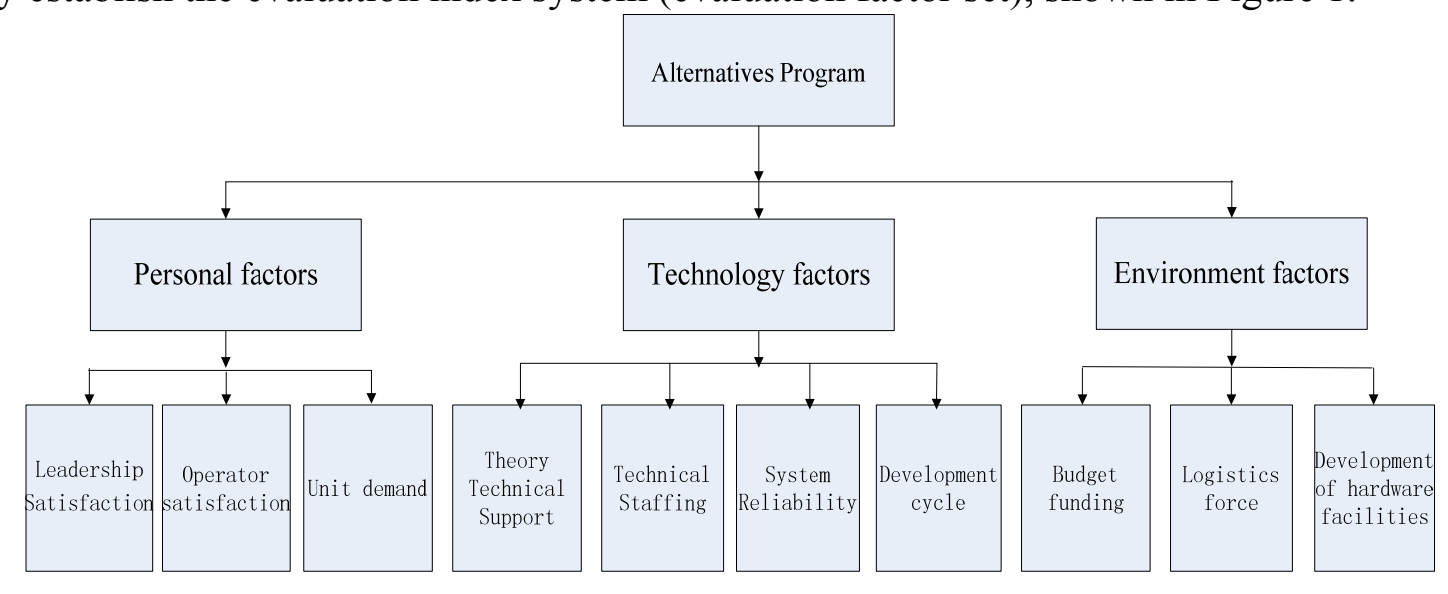

Fig.1 the evaluation index system of the communication equipment simulation training system

The factors set is $U=\left\{U_{1}, U_{2}, U_{3}\right\}, U_{1}=\left\{u_{11}, u_{12}, u_{13}\right\}, U_{2}=\left\{u_{21}, u_{22}, u_{23}, u_{24}\right\}$, $U_{3}=\left\{u_{31}, u_{32}, u_{33}\right\}, U_{i} \cap U_{j}=\varnothing, i \neq j$, So here using two step judge.

Secondly establish the evaluation set and divided the evaluation set into four grades: $V=\{$ excellent, good, general, bad $\}$, that is $V=\left\{v_{1}, v_{2}, v_{3}, v_{4}\right\}$.

Thirdly establish a single factor evaluation. With the first program of semi-physical simulation training system for example, establish one level (single factor) comprehensive evaluation and 
scoring for index system of the alternatives program using expert scoring method, which invited 100 experts scoring for the index system, you can get the judgment matrix, as shown in Table 1.

Table 1 expert judgment Results

\begin{tabular}{|l|c|c|c|c|}
\hline & excellent & good & general & bad \\
\hline Leadership Satisfaction & 20 & 30 & 40 & 10 \\
\hline Operator satisfaction & 35 & 30 & 20 & 15 \\
\hline Unit demand & 40 & 25 & 20 & 15 \\
\hline Theory Technical Support & 30 & 35 & 15 & 20 \\
\hline Technical Staffing & 25 & 30 & 20 & 25 \\
\hline System Reliability & 32 & 24 & 18 & 26 \\
\hline Development cycle & 10 & 15 & 45 & 31 \\
\hline Budget funding & 12 & 10 & 34 & 44 \\
\hline Logistics force & 22 & 20 & 43 & 15 \\
\hline Development of hardware facilities & 18 & 13 & 37 & 32 \\
\hline
\end{tabular}

We can obtain single factor judgment matrix respectively from Table 1 as

$$
\begin{gathered}
R_{1}=\left[\begin{array}{llll}
0.20 & 0.30 & 0.40 & 0.10 \\
0.35 & 0.30 & 0.20 & 0.15 \\
0.40 & 0.25 & 0.20 & 0.15
\end{array}\right. \\
R_{2}=\left[\begin{array}{llll}
0.30 & 0.35 & 0.15 & 0.20 \\
0.25 & 0.30 & 0.20 & 0.25 \\
0.32 & 0.24 & 0.18 & 0.26 \\
0.10 & 0.15 & 0.45 & 0.31
\end{array}\right. \\
R_{3}=\left[\begin{array}{llll}
0.12 & 0.10 & 0.34 & 0.44 \\
0.22 & 0.20 & 0.43 & 0.15 \\
0.18 & 0.13 & 0.37 & 0.32
\end{array}\right.
\end{gathered}
$$

$R_{1}, R_{2}, R_{3}$ are corresponding to evaluation factor set $U_{1}, U_{2}, U_{3}$ respectively. Weight vectors of each evaluation matrix can be calculated by the analytic hierarchy process(AHP)

$$
\begin{aligned}
& A_{1}=\left\{\begin{array}{lll}
0.35 & 0.40 & 0.25
\end{array}\right\} \\
& A_{2}=\left\{\begin{array}{llll}
0.25 & 0.22 & 0.35 & 0.18
\end{array}\right\} \\
& A_{3}=\left\{\begin{array}{lll}
0.42 & 0.33 & 0.25
\end{array}\right\}
\end{aligned}
$$

Fourth the corresponding evaluation results can be calculated by weighted average operator.

$$
\begin{aligned}
& B_{1}=A_{1} \cdot R_{1}=\sum_{i=1}^{4} a_{j i} r_{j i}=\left\{\begin{array}{llll}
0.310 & 0.287 & 0.270 & 0.133
\end{array}\right\}, \quad j=1 \\
& B_{2}=A_{2} \cdot R_{2}=\sum_{i=1}^{4} a_{j i} r_{j i}=\left\{\begin{array}{llll}
0.260 & 0.263 & 0.231 & 0.258
\end{array}\right\}, \quad j=2 \\
& B_{3}=A_{3} \cdot R_{3}=\sum_{i=1}^{4} a_{j i} r_{j i}=\left\{\begin{array}{llll}
0.168 & 0.141 & 0.377 & 0.314
\end{array}\right\}, \quad j=3
\end{aligned}
$$

Fifth establish the second step comprehensive evaluation.

We can obtain the weight vector of secondary evaluation matrix also using the analytic hierarchy process, and then the evaluation results of one step comprehensive evaluation as the evaluation matrix of secondary step evaluation, which can calculate the final evaluation results $B$, as follows:

$$
B=A \cdot R=A \cdot\left[\begin{array}{l}
B_{1} \\
B_{2} \\
B_{3}
\end{array}\right]=\left\{\begin{array}{lll}
0.26 & 0.42 & 0.32
\end{array}\right\}\left[\begin{array}{llll}
0.310 & 0.287 & 0.270 & 0.133 \\
0.260 & 0.263 & 0.231 & 0.258 \\
0.168 & 0.141 & 0.377 & 0.314
\end{array}\right]=\left\{\begin{array}{llll}
0.22 & 0.23 & 0.29 & 0.24\}
\end{array}\right\}
$$


The final evaluation results of the program of physical simulation training systems is general, which can be calculate by the principle of maximum degree of membership.

Sixth quantify the evaluation results.

$E_{1}=\sum_{i=1}^{4} b_{i} v_{i}=59.8$

Similarly, other evaluation results of design programs can get: Evaluation results of program 2( semi-physical + digital technology simulation training systems) is excellent, quantization value as

$E_{2}=\sum_{i=1}^{4} b_{i} v_{i}=86.7$

Evaluation results of program 3(simulation training systems based on visual technology) is good, quantization value as

$E_{3}=\sum_{i=1}^{4} b_{i} v_{i}=77.4$

Evaluation results of program 4(simulation training systems based on virtual reality technology) is good, quantization value as

$E_{4}=\sum_{i=1}^{4} b_{i} v_{i}=72.3$

Thus, program 2 should be the best choice.

\section{Conclusion}

Through multi-level fuzzy comprehensive evaluation method, the optimal choice of the multiple alternatives design program will be specifically quantified, and the calculation is simple and clear, to provide a theoretical basis and data support for the final decision. This method can provide a strong theoretical support for the various subsystems of the design selection and demonstration of communication equipment simulation training systems.

\section{References}

[1] Shi Hui-ling, Ma Jun, Zou Feng-yi. Software Dependability Evaluation Model Based on Fuzzy Theory[A]. International Conference on Computer Science and Information Technology,2008

[2]Liu Jianguo, Bi Jinnan.Introduction to Communication Equipment Support[M]Military Science Publishing House, 2006.

[3]Guo Qisheng,Yang Ruiping.Introduction to Equipment Evaluation[M].北京:National Defense Industry Press, 2005. 